Proceedings of the $2^{\text {nd }}$ ICEENG Conference, 23-25 Nov. 1999
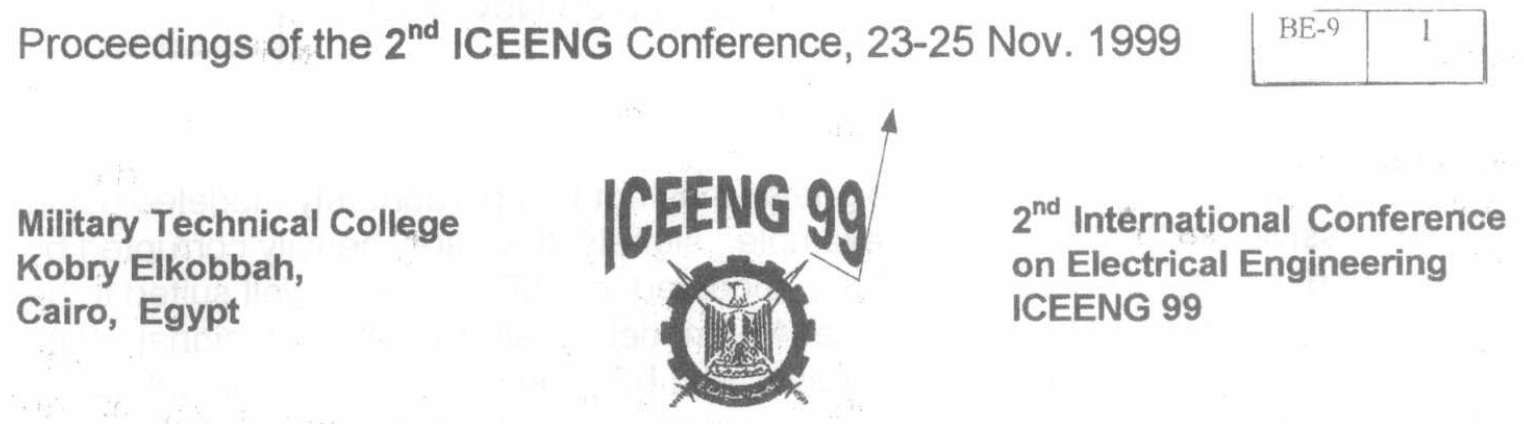

\title{
MODELING OF EGG SIGNALS USING ARMA COEFFICIENTS
}

\author{
Shouman E. I. Shouman*
}

\section{ABSTRACT:}

The Electrogastrogram (EGG) is a new diagnostic tool for the stomach of the human being. Its first measurement was done 75 years ago. At first, the analysis of the EGG signals was very difficult, since the pattern of this signal shows no meaning of the stomach diseases or state. In this work, we try to represent the Stomach State using the Autoregressive Moving Average (ARMA) Coefficients to be able in a later stage to discriminate between different stomach states (Hunger, Digestion, Before, and After Haemodialysis). Three Off-Line ARMA Coefficients estimators and an adaptive one are discussed. These techniques are applied to real EGG signals acquired cutaneously from the abdomen of the human being in one of the army hospitals. Their performances are tested and evaluated to elect one of them for later applications. The results show the efficient accuracy of the recommended algorithm.

\section{KEYWORDS:}

Elecgastrogram, Haemodialysis, ARMA, Adaptive Filter

*Egyptian Armed Forces 


\section{INTRODUCTION:}

In some applications, biomedical signals may not be appropriately modeled using Autoregressive (AR) models. For example, signals that are heavily corrupted by background noise, are not adequately modeled by AR but are well suited to be analyzed by the ARMA models. The AR model is appropriate for signals with frequency responses that have sharp peaks and MA works better on deep valleys.

Several researchers have proposed methods for estimating the ARMA model Coefficients without the need of determining the Moving Average (MA) Coefficients. However, these techniques don't guarantee a nonnegative spectral estimate and sometimes lead to invalid estimate [1].

\section{PRINCIPLES OF THE ARMA MODEL:}

The input Signal, which supposed to be any random stream of numbers, is introduced to the ARMA Model to get the output signal. The structure of the AFiMA Model is shown in Fig. 1.

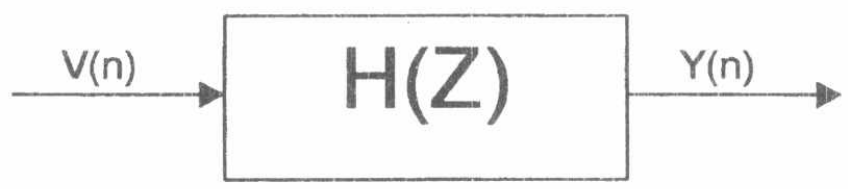

Fig.1. Structure of the ARMA Model

While the transfer function of the ARMA model have the form in equation (1):

$$
H(z)=\frac{\sum_{i=0}^{q} b_{i}^{*} Z^{-i}}{1+\sum_{i=1}^{p} a_{i} * Z^{-i}}
$$

Where:

$V(n)$ : is the input of the ARMA Model, which may be White Gaussian Noise (WGN), Uniform, ... etc. of Random Numbers.

$Y(n)$ is the output form the ARMA Model, which corresponds to the EGG signal.

$b_{i}$ : are the coefficients of the MA Model.

$a_{\mathrm{i}}$ : $\quad$ are the Coefficients of the AR Model.

$p, q$ : $\quad$ are the orders of the AR and MA Models respectively $(p \geq q)$.

The previous transfer function must satisfy the stability conditions, i.e. all the poles of the AR Model must lie within unit circle of the Z-plane. The evaluation will include calculations of the Signal-to-Noise Ratio (SNR) before and after applying the AFiMA Model, and the efficiency $(\zeta \%)$ of the algorithm: 


$$
\begin{aligned}
& S N R=20 * \frac{\log (\max (|Z|)}{\sigma^{2}} \\
& \zeta \%=\frac{S N R(\text { afterARMA })-S N R(\text { beforeAMRA })}{\operatorname{SNR}(\text { before } A R M A)} * 100
\end{aligned}
$$

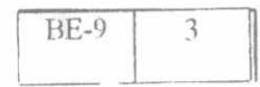

Where:

$|Z|: \quad$ is the magnitude response of the signal, for which SNR is calculated.

$\sigma^{2}: \quad$ is the variance of this signal.

\section{RECORDING OF EGG SIGNALS:}

Two experiments were conducted on healthy and unhealthy groups. The first one studied the changes of EGG $[6,7]$ from healthy persons under different conditions (Hunger and Digestion), eleven adults (men) with ages between 21 and 25 years were selected to be volunteers in our experiment. The main factor of choice was that they are free from any disease related to the digestive system. Moreover, they do not take any pharmacological component to activate the appetite. The second experiment studied the influence of Haemodialysis procedure on stomach electrical activity, ten adults (men) with ages between 35 and 70 years suffering from renal failure and they have to perform Haemodialysis process three times per week. Silver-Silver-Chloride surface electrodes type were used to pick up the biopotential over stomach $[8,9]$. The electrode is fixed on a location far $3 \mathrm{~cm}$ from the line between the umbilicus and the xiphoid process, with the ground terminal fixed on the bottom of the left leg.

The recording were made for $20 \mathrm{~min}$. with sampling rate of $2 \mathrm{~Hz}$ for all the volunteers. The Recording were done after an hour of eating a standard meal. We have developed the required software to acquire the data using the data acquisition board DAQ-12 and a 486DX2-66 PC.

\section{CALCULATION OF MODEL PARAMETERS:}

Three algorithms have been implemented to calculate the ARMA Model parameters. The performance of each algorithm is thoroughly studied in order to choose the best algorithm to represent the EGG signals.

\section{a. Algorithm I:}

The steps of this algorithm [1] is shown in Table 1: 
Table 1. Algorithm I of the ARMA Modeling

$$
\begin{aligned}
& \text { Input Max AR Model order ( } \mathrm{p} \text { ) } \\
& \text { Remove DC level from the input signal ( } \mathrm{x} \text { ) } \\
& \text { Calculate AR Coefficients (a) with order } \mathrm{p} \text {, using }(\mathrm{x}) \\
& e_{t}=\sum_{k=0}^{p} a_{k} X_{t-k}, \quad d_{t}=\sum_{k=0}^{p} a_{k} X_{t+k} \\
& \text { For } \mathrm{q}=1 \text { to } \mathrm{p} \\
& \quad W_{k}=1-\frac{k}{q+1} \\
& \quad b_{k}=W_{k} \frac{1}{N-p}\left[\sum_{t=p+1}^{N-k} e_{t} e_{t+k}+\sum_{t=1}^{N-p-k} d_{t} d_{t+k}\right] \quad k=0,1, \ldots, q \\
& \quad b_{k}=b_{-k} \\
& \text { Applying the ARMA Model on the input (v) and get the output (y) } \\
& \text { Calculate Mean Square Error (MSE) } \\
& \text { End-For } \\
& \text { Select the b 's coefficients with order q corresponding to min. MSE }
\end{aligned}
$$

From the performance analysis [2], it was found that the noise level existed in the signal affects the order of the AR model ( $p)$. In other words, $(p)$ increases as noise level increases. Also, (p) mainly depends on the number of frequencies included in the signal ( 2 AR coefficients are required for each 1 dominant frequency in the signal). The efficiency $(\zeta \%)$ is better when using uniform noise distribution than the WGN, for free and low noise level. But when using the uniformly distributed noise, it produces inadequate results for high noise level, unlike the WGN. So, WGN is the best choice as an input to the algorithm $v(t)$.

\section{b. Algorithm II:}

The new algorithm is based on the technique developed by Durbin to calculate the MA model [3]. The steps of this algorithm are shown in Table 2:

Table 2. Algorithm II of the ARMA Modeling

Input Max AR Model order ( $p)$

Remove DC level from the input signal $(x)$

Calculate AR Coefficients (a) with order $p$, using $(x)$

Choose $L_{a a}$ such that: $N>L_{a a}>2 q \quad N$... number of samples in the input signal

For $q=1$ to $p$

$$
\begin{array}{ll}
\left(R_{a a}\right)_{i j}=\frac{1}{L_{a a}+1} \sum_{m=0}^{L_{a a}-|i-j|} a_{m} a_{m+|i-j|}, & i, j=1,2, \ldots, q \\
\left(P_{a a}\right)_{i}=\frac{1}{L_{a a}+1} \sum_{m=0}^{L_{a a}-i} a_{m} a_{m+i}, & i=1,2, \ldots, q \\
b=-R_{a a}{ }^{-1} * P_{a a} &
\end{array}
$$

Applying the ARMA Model on the input (v) and get the output (y) Calculate MSE

End-For

Select the b 's coefficients with order q corresponding to min. MSE 
The performance of this algorithm [2] is tested. It has been found that WGN is suitable as an input to the algorithm for almost all levels of noise, so WGN can be used as the best choice. It can be seen that as noise level increased, the algorithm decreased the SNR of the signal after applying the ARMA model. Then, this algorithm is not suitable in case of noise existence.

This algorithm produces bad results in case of large number of frequencies dominant in the input signal. Then, this algorithm is not suitable with the EGG signals since they contain a considerable number of frequencies with certain level of noise.

\section{Algorithm III:}

This algorithm is based on estimating the transfer function of the input signal with (AR order $=$ MA order) [4]. The steps of this algorithm are shown in Table3:

Table 3. Algorithm III of the ARMA Modeling

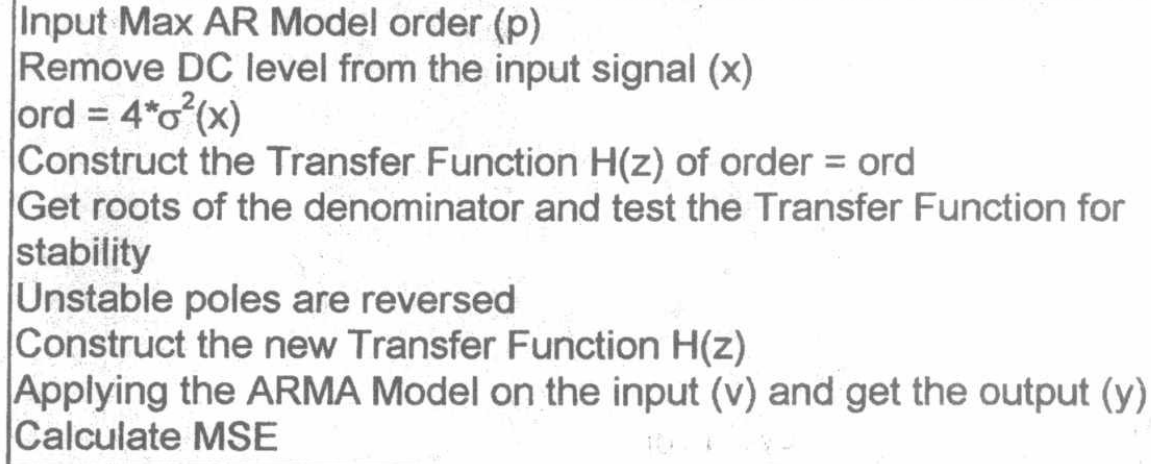

The performance of the algorithm is analyzed [2]. It has been found that the algorithm produces better results in case of high noise level, when introducing uniform input. On the other hand, WGN as input produces better results in case of free noise signals or signals with small noise level. It has been found that, this algorithm is suitable for signals containing medium number (2-4) of dominant frequencies.

Finally, we can conclude the analysis results of the performance of the previous three algorithms as follows. Firstly, second algorithm fails to identify the frequencies in case of low and high noise levels, also in case of dominant frequencies greater than 2 frequencies. Secondly, comparing the factor $(\zeta \%)$ for both first algorithm, and third algorithm, we can get the following. Firstly, w. r. t. effect of noise level, when WGN input is introduced to the first algorithm, it showed better results for noise free and low noise level. But in case of high noise level third algorithm shows better results. Secondly, w. r. t. effect of number of frequencies, first algorithm shows better results for low and high number of frequencies, and third algorithm shows better results for medium (2-4 dominant frequencies) number of frequencies.

Since, the EGG signals is expected to have high level of noise (SNR is low), and the number of useful dominant frequencies in the signal is medium. So, the suitable algorithm to be used is the third algorithm. 


\section{RESULTS:}

The ARMA coefficients of the Normal group of EGG signals in both states (Hunger and Digestion) are calculated using the third algorithm. Then trying to discriminate between these two states with either the numerator or the denominator, it has been found that the denominator coefficients give bad discrimination. On the other hand, only coefficient number 2 and 4 of the numerator coefficients (b2 and b4) give approximately good discrimination, as in Fig.2. As seen in figure, it succeeded in the discrimination process with accuracy of $87.5 \%$ in Hunger state, and with accuracy $88.75 \%$ in Digestion state, and succeeded with accuracy of $93.75 \%$ for the Before state, and with accuracy of $90 \%$ for After state.
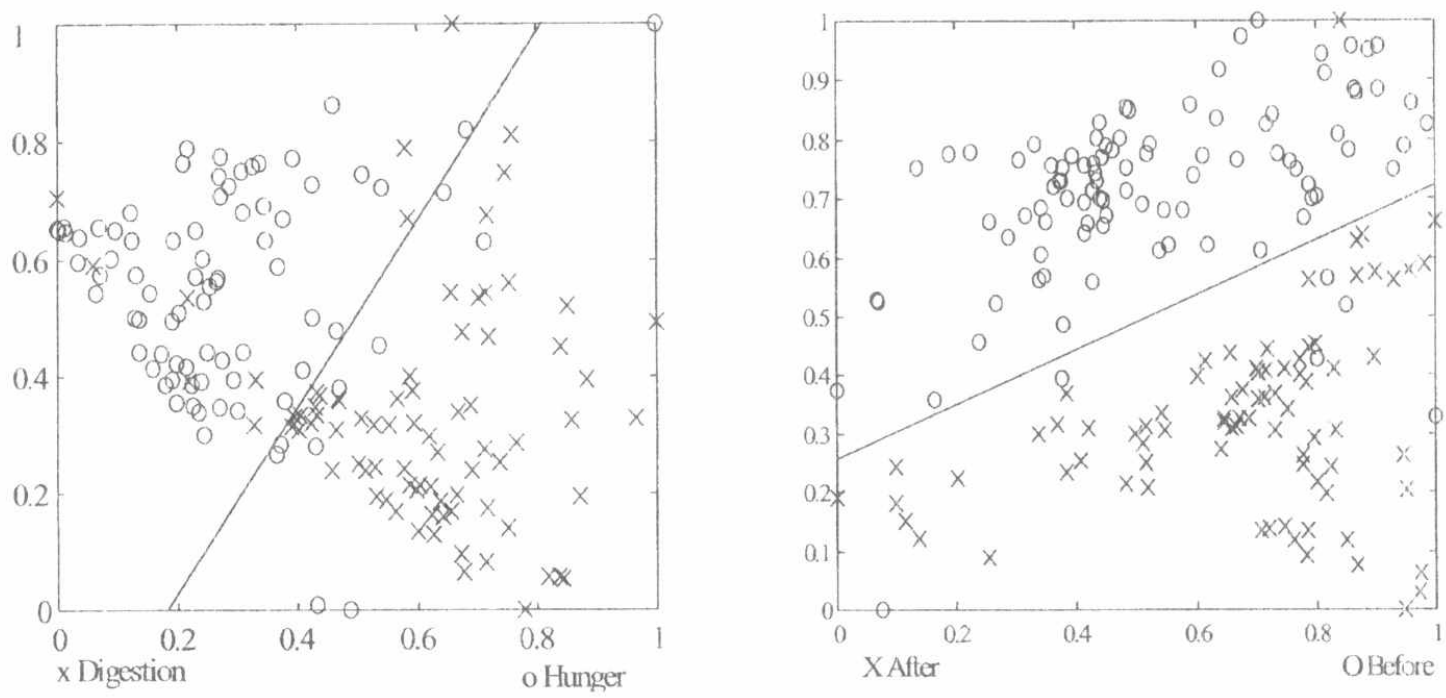

Fiq.2. Scattered Diagram between (b2,b4)

Trying to increase the accuracy of discrimination between the stomach states, we introduce an adaptive ARMA Coefficients Estimator [2], as in Table 4. The Block diagram of the Adaptive ARMA Model [5] is illustrated in Fig.3.

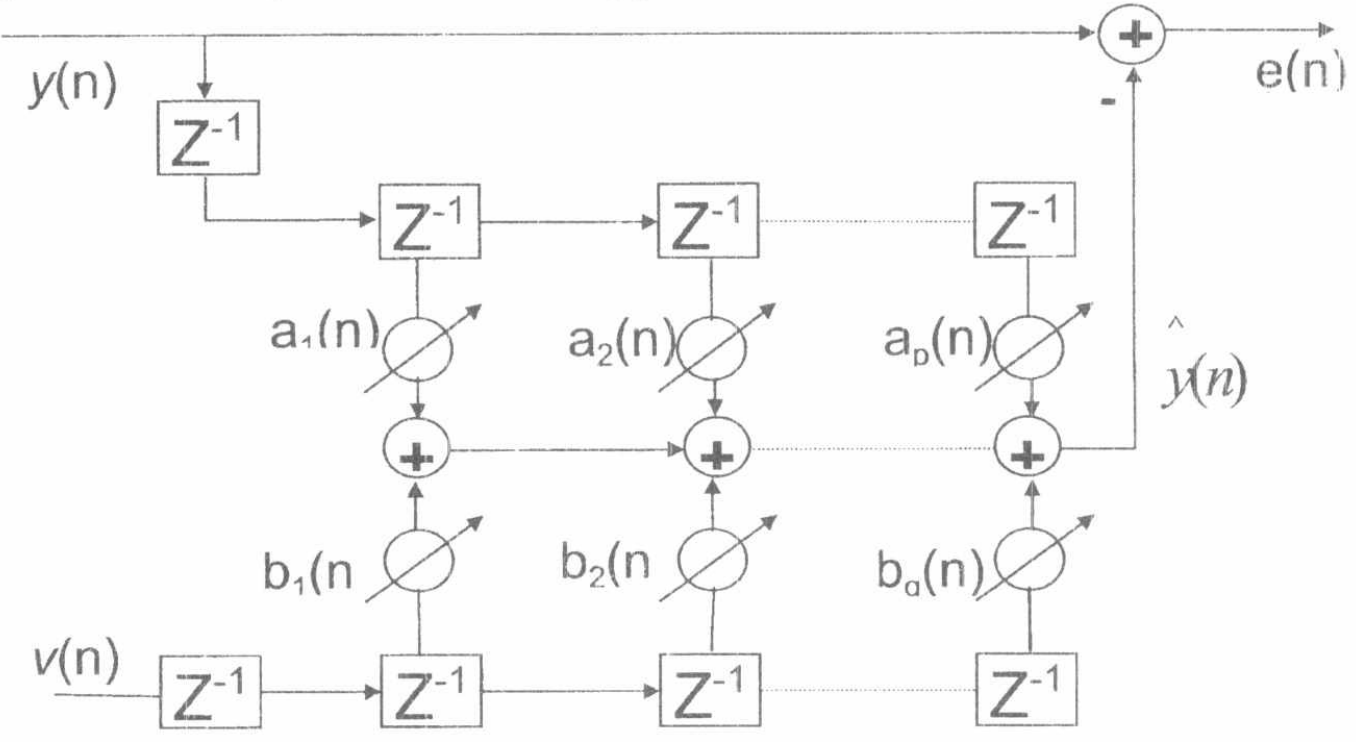

Fig.3. Adaptive ARMA Technique 
Proceedings of the $2^{\text {nd }}$ ICEENG Conference, 23-25 Nov. 1999

\begin{tabular}{|l|l|}
\hline BE-9 & 7 \\
\hline
\end{tabular}

Where:

e(n) The Error between the estimated signal and the EGG signal.

$v(n) \quad$ The WGN input to the Adaptive ARMA Model.

$y(n) \quad$ The EGG signal.

$\wedge$

$y(n) \quad$ The estimated signal.

$a_{m} \quad$ The AR coefficients, $(m=1,2, \ldots, p)$.

$b_{m} \quad$ The MA coefficients, $(m=1,2, \ldots, q)$.

It is assumed that the input to the system is White Gaussian Noise WGN ( $v$ ), and the output is the measured signal $(y)$. Trying to find the ARMA model Coefficients (a's , and b's) that can represent the EGG signal $(\hat{y})$. The Coefficients a's and b's are calculated adaptively using the algorithm shown in Table 4.

Table 4 Algorithm of Adaptive ARMA

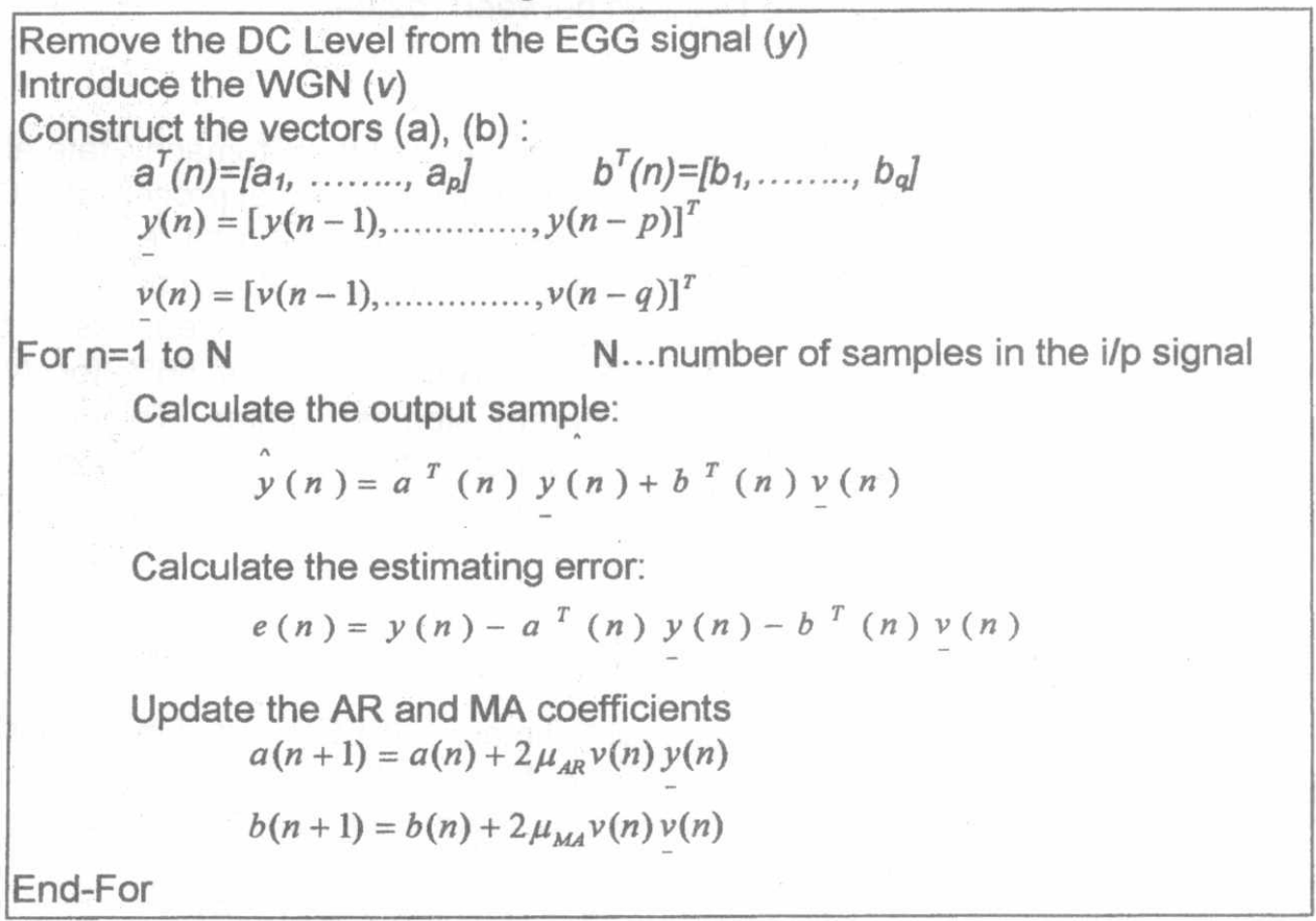

The performance of the algorithm is analyzed from the previous factors point of view. From the analysis results, it has been found that this algorithm shows better performance than the previous algorithm (Off-Line Techniques).

The resultant coefficients are normalized, and then they are plotted on a scatter diagram. Attempting to make discrimination between the Hunger and Digestion states of the stomach: It has been found that the best parameters that can discriminate these two states are the coefficients of the Moving Average process (MA) number 2 and 4 (b2, b4), as shown in Fig.4. From this figure, it can be seen that the algorithm succeeded to discriminate between the Hunger and Digestion with accuracy of $87.5 \%$ for Hunger and of $91.25 \%$ for Digestion, and with an accuracy of $97.56 \%$ for the Before state, and an accuracy of $87.8 \%$ for the After state. 

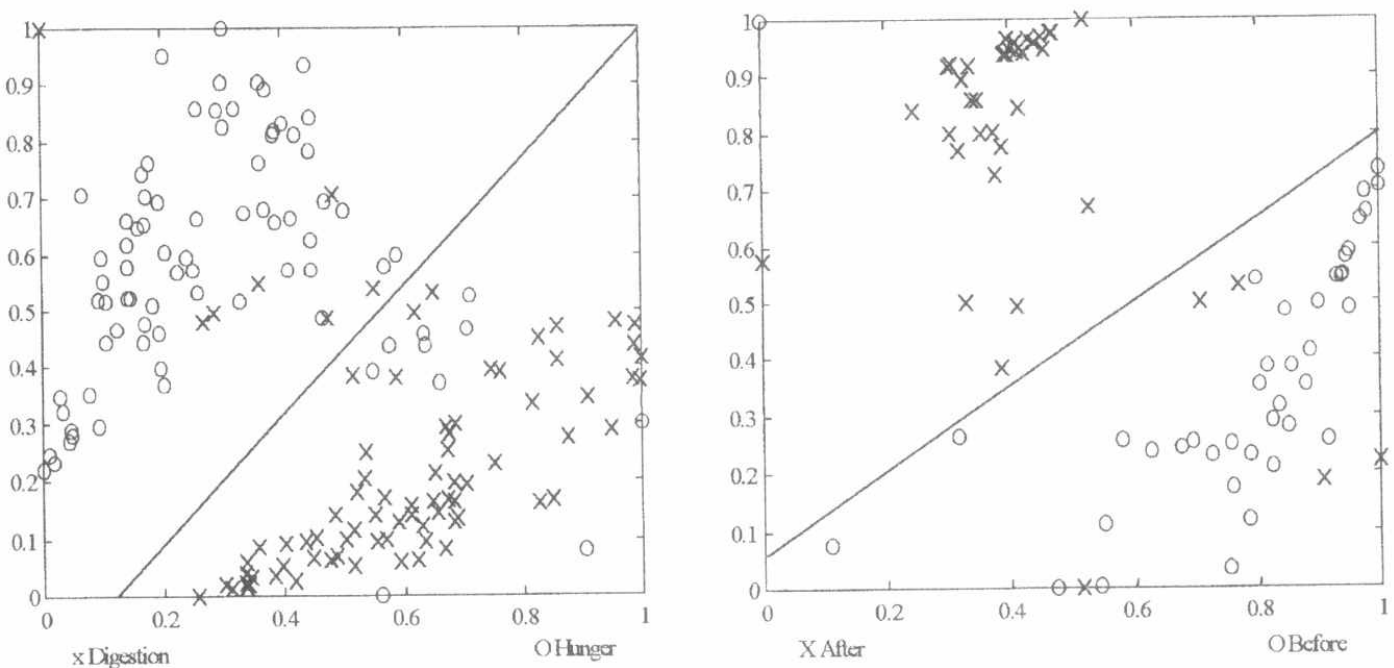

Fig.4. Scattered Diagram between (b2,b4)

\section{CONCLUSION:}

The Electrogastrogram can be used as a representation of the Stomach State after applying the signal processing techniques. The two types of signal processing: OffLine (Acquisition of the EGG signal, then processing and extract features) and OnLine (processing of the EGG signal during the acquisition process) can be used with the EGG signals. The adaptive technique produces high accuracies (reaches $87.5 \%$ for Hunger and Digestion states, $91.25 \%$ for Before, and $97.56 \%$ for After Haemodialysis) of discrimination between different stomach states. This is true, since the EGG signals are nonstationary signals. Estimated ARMA Coefficients can be used to represent the Stomach State qualitatively.

\section{REFERENCES:}

[1] Steven M. Kay, "A New ARMA Spectral Estimator", IEEE Trans. On Acoustics, Speech, and Signal Processing, Vol. ASSP-28, No.5, October (1980).

[2]Elsamahy, E., "A Computer-Based System for Measurements and Processing of Special Signals", M.Sc. Thesis, (1998).

[3] Akay, M., "Biomedical Signal Processing", Academic press., (1994).

[4] MathWorks, "Signal Processing Toolbox", (1996).

[5]Chen, J., Vandewalle J., "Adaptive Spectral Analysis of Cutaneous

Electrogastrogram signals using Autoregressive Moving Average Moedling", Med. Biol. Eng. Computer, Vol. 28, pp. 531-536,(1990).

[6]Alvarez, W. C.,"The Electrogastrogram and what it shows", J. Amer. Med. Assoc., Vol. 8, pp. 1116-1118,(1992).

[7]Stren, R. M., and Koch, K. L., "Electrogastrography: methodology, validation and application", Prager, New York, (1985).

[8]Chen, J., et al., "Clinical Applications of Electrogastrography", AJG, Vol. 88, No.9,pp. 1324-1336, (1993)

[9]Chen, J., Mccallum R. W., "Electrogastrogram: Measurement, analysis and prospective applications", Med. Biol. Eng. Computer, Vol. 29, pp. 339-350, (1991). 